\title{
Lettres, chiffres et dessins : sur un ostracon énigmatique conservé à la BNU
}

\section{Régine Hunziker-Rodewald}

\section{(2) OpenEdition}

1 Journals

Édition électronique

URL : http://journals.openedition.org/rbnu/1476

DOI : 10.4000/rbnu.1476

ISSN : 2679-6104

Éditeur

Bibliothèque nationale et universitaire de Strasbourg

\section{Édition imprimée}

Date de publication : 1 mai 2016

Pagination : 16-23

ISBN : 9782859230623

ISSN : 2109-2761

\section{Référence électronique}

Régine Hunziker-Rodewald, "Lettres, chiffres et dessins : sur un ostracon énigmatique conservé à la BNU », La Revue de la BNU [En ligne], 13 | 2016, mis en ligne le 01 mars 2020, consulté le 12 décembre 2020. URL : http://journals.openedition.org/rbnu/1476 ; DOI : https://doi.org/10.4000/rbnu.1476

\section{(c) (i) (2)}

La Revue de la BNU est mise à disposition selon les termes de la Licence Creative Commons Attribution - Pas d'Utilisation Commerciale - Partage dans les Mêmes Conditions 4.0 International. 


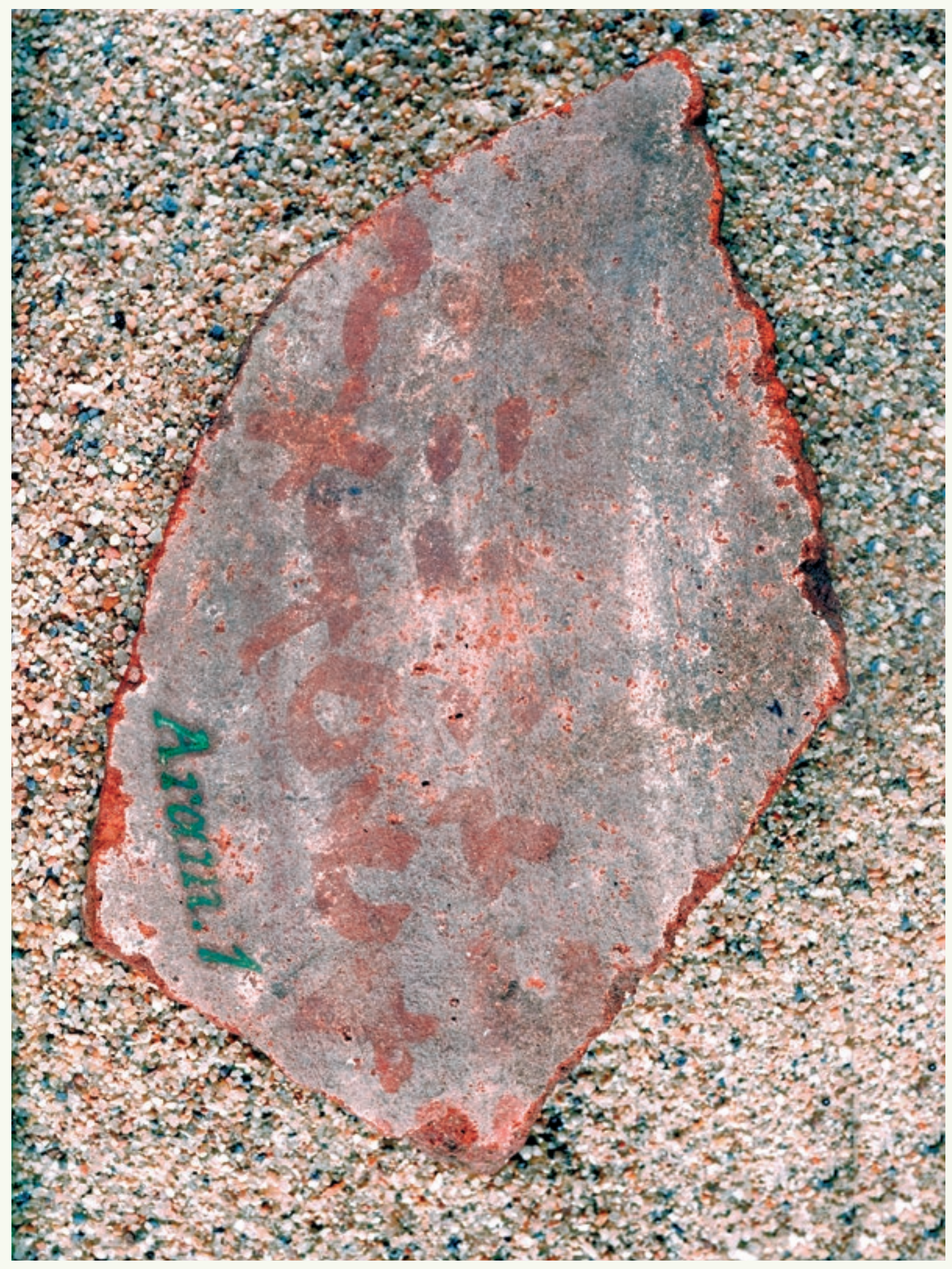




\section{LETTRES, CHIFFRES ET DESSINS : SUR UN OSTRACON ÉNIGMATIQUE CONSERVÉ À LA BNU}

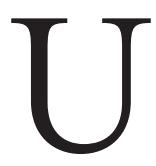

$\mathrm{n}$ ostracon est un tesson de poterie qui, dans les civilisations antiques de l'Égypte, du Levant et de la Grèce était utilisé, après qu'un récipient en argile s'était cassé, comme support bon marché d'écriture ou de dessin. Parfois on désigne également par ce terme un éclat de calcaire inscrit ou décoré. Ce sont en tous les cas des produits de rebut présents en quantité massive, qui pouvaient servir à tout instant du quotidien de surface disponible pour des notes éphémères, des reçus, des messages courts, des bulletins de vote, un bref rapport, des listes ou des lettres. Dans la plupart des cas, l'inscription se faisait à l'encre, à l'aide d'un calame, ou, moins souvent, par incision. L'encre appliquée était généralement noire ou brun foncé, assez rarement de couleur rouge.

L'ostracon dont il s'agit dans le présent article est un tesson de 7 à $10 \mathrm{~cm}$, d'une épaisseur qui varie entre o,8 et $0,9 \mathrm{~cm}$. L'argile est de qualité sablonneuse et de couleur rougeâtre. La surface externe du tesson, légèrement convexe, a été lissée à l'aide d'un outil qui a laissé de fines rayures et a été couverte, après la cuisson, d'un engobe blanc partiellement abîmé. Sur la surface interne, légèrement concave, en brun-rouge pâli, les rainures des doigts de l'artisan qui a produit le récipient original sur son tour de potier sont toujours visibles. Le tesson est brisé sur tous ses côtés, ici et là la ligne de cassure est marquée de petits éclats.

Le recto du tesson, la surface légèrement convexe donc, comporte plusieurs signes alignés à l'encre rouge avec un calame assez épais, ce qui donne à l'inscription une apparence relativement grossière. Certaines parties de quelques signes sont abîmées ou presque effacées. Le verso comporte dans le coin supérieur droit, sur l'original difficilement identifiable, le dessin pâli d'un quadrupède et d'un homme assis, l'un au-dessus de l'autre. Sur des photographies en haute résolution, un crochet d'angle rectangulaire, dont l'aile horizontale est dirigée vers la gauche, est visible directement derrière l'animal et derrière l'homme assis.

Les deux faces du tesson portent différents numéros d'inventaire : au recto, dans le coin inférieur droit, de couleur verte, l'indication Aram.1 qui atteste d'une première classification. Notre ostracon avait donc été associé au groupe des six ostraca araméens conservés à la $\mathrm{BNU}{ }^{1}$. Au verso, deux marques ont été ajoutées : dans le coin inférieur gauche, en noir, dans une écriture ornée et différente de celle du recto, l'indication Aram.1 et au bord supérieur le numéro 1298 . Une troisième marque disposée tête-bêche et effacée intentionnellement est le numéro 533 (ou, moins probablement, 553). Aujourd'hui cet objet porte la cote « ostracon 1298 ».

\section{L'ostracon 1298 et son histoire récente}

D’après une note manuscrite de l'égyptologue Wilhelm Spiegelberg dans l'inventaire allemand de l'Institut d'égyptologie de l'Université de Strasbourg, l'ostracon 1298 provient des fouilles archéologiques de son confrère anglais James Edward Quibell pendant l'hiver 1895-96 dans le Ramesseum, le temple funéraire de Ramsès II. Ayant été trouvé dans les environs du Ramesseum ("Umgebung des Ramesseums "), ce débris épais recouvert d'une écriture rouge non égyptienne (" dicker Scherben mit roter unaegyptischer Schrift ") a été offert par l'archéologue même à l'Institut d'égyptologie et 
a ensuite, entre le 2 mars 1901 et le 24 janvier 1902, été transféré à la Kaiserliche Universitäts- und Landesbibliothek (l'ancêtre de l'actuelle BNU) ${ }^{2}$. L'ostracon 1298 est arrivé à la bibliothèque dans un lot de huit tessons à qui l'on a attribué les numéros d'inventaire 1298 à 1305 . Sept des huit ostraca, les nos 1299 à 1305, sont issus d'une série avec une écriture hiératique de grand module, à l'encre noire, sur une poterie fine, de couleur beige claire et d'une épaisseur de 0,5 à $0,6 \mathrm{~cm}$. Entretemps, ils ont tous été publiés ${ }^{3}$, l'ostracon 1298 restant seul inédit et rangé parmi les ostraca araméens. Ces derniers ont eux aussi tous été publiés en 1915 (cf. note 1), sauf le tesson 1298 ! Car jusqu'à présent, toutes les tentatives visant à le déchiffrer n'ont pu aboutir.

Plus de 100 ans après son arrivée à Strasbourg, le 20 octobre 2009, nous avons examiné d'un regard neuf cet ostracon énigmatique et, à cette occasion, nous avons décelé au verso du fragment la présence du dessin d'un quadrupède et d'un homme assis, qui ressemble à un signe hiéroglyphique. Ces dessins étaient jusque-là restés ignorés : nulle mention de ces figures ni dans le carnet d'inventaire de l'Institut d'égyptologie ni dans le répertoire de la bibliothèque. Même la personne qui a ajouté le $\mathrm{n}^{\circ} 1298$ sur le verso n'avait pas remarqué ces images : le chiffre touche presque le côté arrière de l'animal et se superpose pleinement au signe d'un crochet d'angle rectangulaire qui a été ajouté derrière le dessin du quadrupède.

En janvier 2010, en tant que responsable du Groupe de recherches et d'études sémitiques anciennes (GRESA) de la Faculté de théologie protestante de l'Université de Strasbourg, nous avons déposé un projet de recherche et une demande de soutien financier auprès du Conseil scientifique de l'université pour l'analyse paléographique, le classement historique et - enfin ! - la publication de l'ostracon 1298. Par la suite, en septembre 2010, des photos numériques du tesson et de nombreux clichés à l'aide de différents filtres ont été réalisés par les photographes de la BNU et de l'Unité mixte de service et de recherche 3227 de l'Université de Strasbourg (MISHA) ${ }^{4}$. $\mathrm{Au}$ cours de nos recherches, nous avons présenté et discuté l'ostracon 1298 en juillet 2011 au King's College à Londres, lors d'une séance du groupe " Epigraphical and Paleological Studies Pertaining to the Biblical World ", organisée à l'occasion de la rencontre annuelle de la Society of Biblical Literature (SBL).

\section{L'inscription de l'ostracon 1298 (recto)}

Les signes à l'encre rouge sur la face recto de l'ostracon 1298 qui, au début du $20^{e}$ siècle, ont été classés par Wilhelm Spiegelberg comme " unaegyptisch " (non égyptiens) restent difficiles à interpréter. Même la direction de lecture, à l'horizontale ou à la verticale, n'est pas sûre. Après avoir examiné les 4958 ostraca, en toutes les langues, qui sont conservés dans les réserves de la $\mathrm{BNU}^{5}$, le tesson 1298 doit être considéré comme unique. Car il pose trop de problèmes pour être lu dans le domaine ouest-sémitique (à quoi correspondait le classement sous la mention "Aram. ") ; aussi a-t-il désormais été changé de tiroir dans la collection et été rangé parmi les ostraca indéterminés.

Voyons maintenant plus précisément l'inscription en lecture verticale, se présentant comme une liste (voir ill. p. 16). L'écriture épaisse des signes reflète une conduite de main relativement hâtive, mais expérimentée. L'alignement suit à peu près les structures effectuées sur la surface du récipient original lors de son lissage avant la cuisson. Or à la ligne 1, la première lettre tout en haut à gauche, forme courbée vers la droite avec un pied qui descend verticalement, sort de l'orientation commune de toutes les lettres suivantes. Elle a probablement été ajoutée après la deuxième lettre $(|x|)$. L'agrandissement montre qu'en exécutant la première lettre, le pinceau a été posé à un demi-millimètre du bord supérieur du tesson. Ce détail indique que la lettre est complète et qu'à cet endroit, la fracture du tesson existait déjà avant la mise en place de l'inscription. De plus, en haut, à la droite de cette même lettre, au bord supérieur de l'ostracon, il n'y a aucune trace d'un autre couple de traits verticaux parallèles, ce qui montre qu'on se trouve au début de la liste présumée.

Lue de gauche à droite, celle-ci consiste en six " groupes " de signes, c'est-à-dire qu'elle se compose sur chaque ligne d'une lettre suivie de chiffres (?). À quatre reprises, il s'agit de deux courts traits verticaux et mis en parallèle (lignes 1 à 4) ; une fois, la lettre droite représente probablement une valeur numérique (ligne 5); enfin, en bas, on trouve de nouveau les deux traits parallèles, en analogie avec ceux d'en haut ou encore (autre lecture possible) appartenant à une sorte $\mathrm{d}^{\prime} / \mathrm{u} /$ large (ligne 6). Tout en bas du tesson, trois petits bouts de signes sont en outre visibles. On peut en conclure qu'à l'origine, la liste était plus longue et qu'à cet endroit-là, l'ostracon a été cassé après la mise en place de l'inscription. Dans chaque lettre, les traits de pinceau relativement épais révèlent 

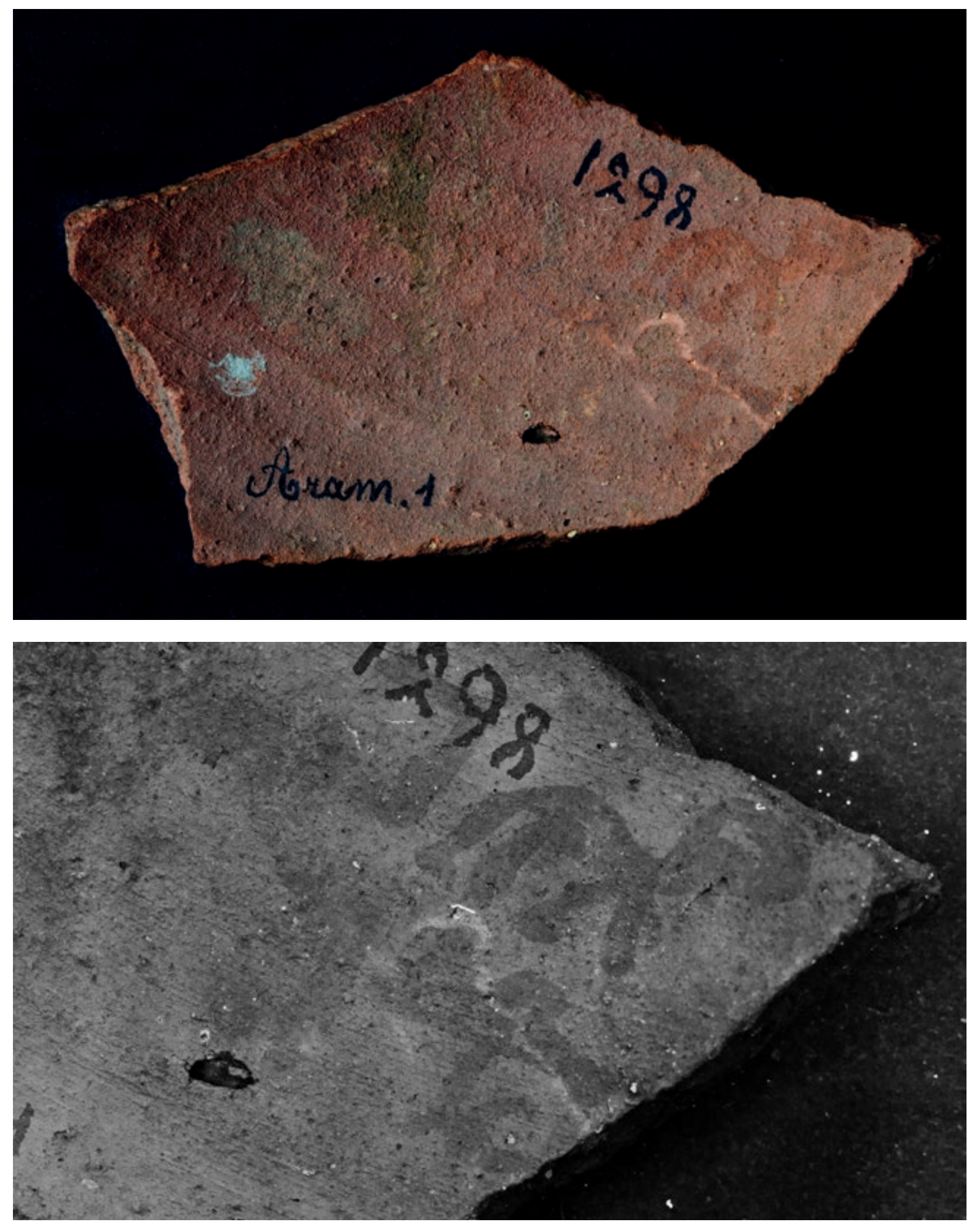

(haut) L'ostracon 1298 (verso) portant le dessin d'un quadrupède et d'un homme assis qui tient une sorte de " mégaphone " (coll. BNU).

(bas) Les dessins du verso de l'ostracon 1298 photographiés en UV (coll. BNU). 


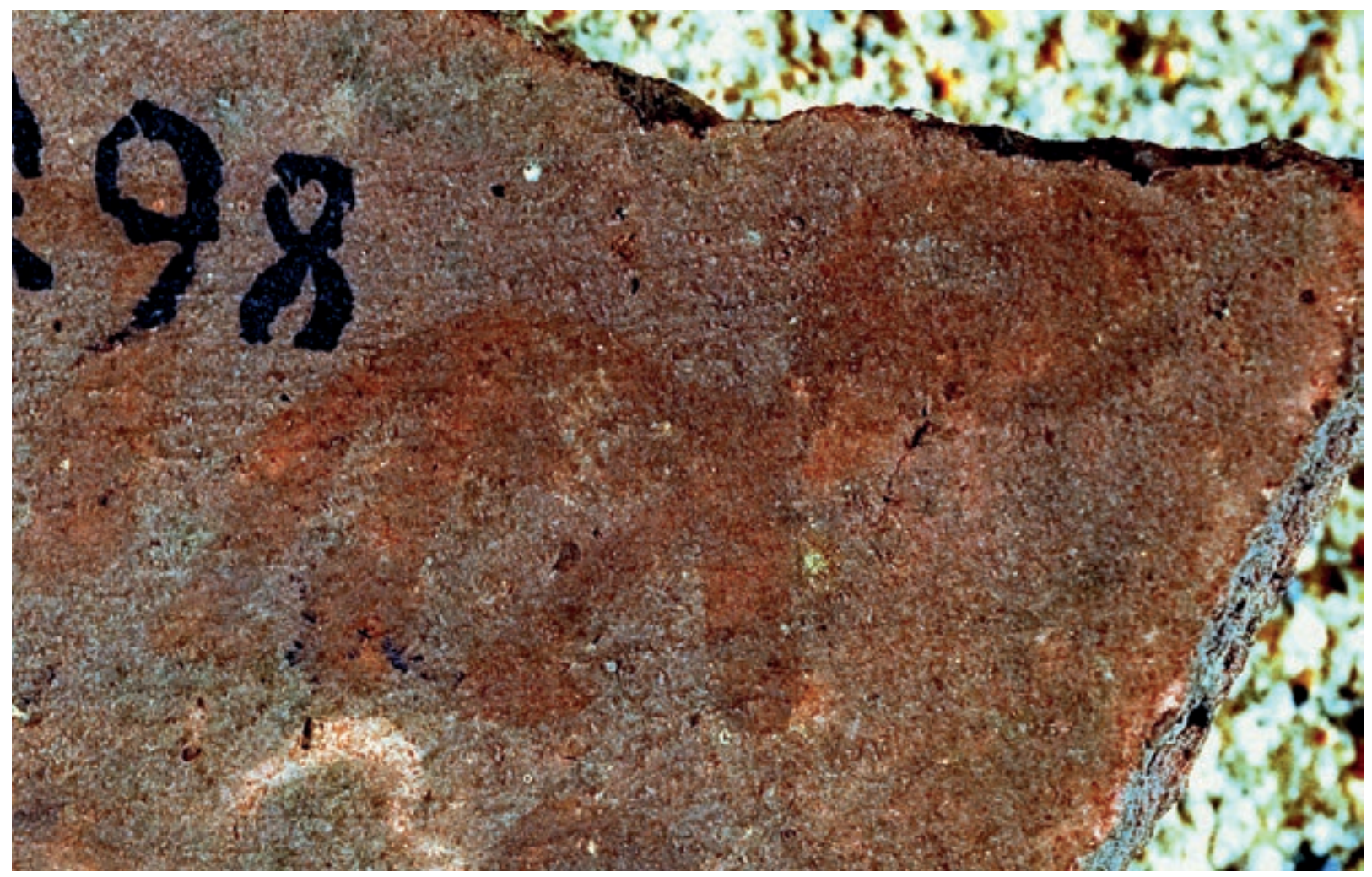

L'« éléphant à la trompe levée »

dessiné au verso de l'ostracon 1298 (coll. BNU). 
une direction d'écriture de haut en bas et de droite à gauche. La seule exception est le deuxième trait, plus large que le premier, de la lettre $|x|$ (ligne 2), pour lequel le pinceau a été mené de gauche à droite. Mais en admettant que l'hypothèse d'une liste de lettres et de chiffres soit correcte, nous achoppons toutefois, jusqu'ici, sur le sens de cette énumération.

$\mathrm{Au}$ cours de ces dernières années, les signes qui figurent sur l'ostracon 1298 ont été examinés intensément par nous-mêmes, en coopération avec des spécialistes à l'échelle internationale dans le domaine des langues ouestsémitiques, du hiératique, du démotique, du méroïtique, du copte et mêmes des marques d'identité dans les listes de main-d'œuvre ${ }^{6}$. Malheureusement, jusqu'à ce jour, ces signes n'ont pu être identifiés dans leur ensemble d'une manière convaincante. Une piste serait de les associer avec une graphie ancienne de lettres coptes (hori, chi, [?], omikron, epsilon, ti) et de chiffres grecs ou démotiques. En disant cela, je passe la barre au futur capitaine !

\section{Les dessins de l'ostracon 1298 (verso)}

Sur le verso de l'ostracon 1298, nous avons repéré deux dessins et deux chiffres (?) ajoutés directement derrière les figures esquissées. À la différence du recto qui a été couvert d'un engobe blanc, lequel fait bien ressortir les signes appliqués à l'encre rouge, la face verso en brunrouge est très pâle et les dessins appliqués, eux aussi à l'encre rouge, ne sont qu'à peine visibles. Or grâce à des traitements photographiques, notamment par différents filtres, un quadrupède avançant vers la droite et un homme assis et " regardant " lui aussi vers la droite ont apparu.

Le quadrupède atteste du même trait de pinceau et donc de la même main de scribe que les lettres de la face recto. Il s'agit très probablement d'un éléphant ayant levé la trompe au-dessus de sa tête, d'après un geste caractéristique connu comme " douche d'eau " ou "douche de poussière ". Avec une silhouette svelte, des pattes longues et avec une queue peu visible, ce type d'éléphant a des épaules ténues, un front renflé vers le haut et une trompe partiellement trop grosse. On devine peut-être même le dessin d'une défense. La ligne du dos est concave, le ventre descend d'entre les jambes de devant vers les pattes de derrière. Ces dernières sont presque parallèles, tandis que les pattes de devant forment une sorte de large $\mathrm{V}$ inversé. À l'extrémité de trois des pattes, une partie élargie indique le pied. Dans la région du cou (très fin), à droite, une tache en rouge dégradé peut être interprétée comme le bout d'une oreille. Derrière l'éléphant, partiellement couvert par le numéro 1298, un crochet d'angle rectangulaire dirigé vers la gauche rappelle le chiffre 5 en hiératique.

Au-dessous de l'éléphant, un homme, dirigé vers la droite, est assis. Ce dessin est presque invisible à l'œil nu. Il s'agit d'un homme dessiné comme d'après un modèle hiéroglyphique. Il a un genou plié, de telle sorte que le pied est posé sur le sol. Un de ses bras est replié vers l'arrière et tient en même temps une sorte de trompette en forme de pavillon, s'élargissant vers la droite et posée à sa bouche. De semblables trompettes, plus fines, avec un long cou, ont été retrouvées dans la tombe de Toutânkhamon. La figure de l'homme est très proche de la ligne de cassure du tesson, à peu près à la hauteur de la première lettre de la liste du recto. Derrière ce joueur d'instrument à vent, il y a peut-être les traces d'un autre crochet d'angle rectangulaire dirigé vers la gauche, mais la barre horizontale est plus courte et la forme légèrement différente.

Comme les signes sur le recto, les deux dessins du verso et leurs rapports mutuels restent également énigmatiques. L'attitude de l'éléphant, la trompe levée, est-elle une réaction au son sorti de la trompette ? Quel est en outre le rapport entre les dessins et les " chiffres" ajoutés derrière ? Un autre problème est celui du rapport entre la liste supposée du recto et les dessins du verso, qui évidement ont été faits par le même " artiste ".

\section{L'éléphant de l'ostracon dans son contexte historique}

À cause de la ligne du dos, de la relativement petite tête et du bout protubérant de l'oreille le long du cou, l'éléphant représenté sur le verso de l'ostracon 1298 s'avère comme un représentant de l'espèce africaine. Même si l'ostracon a été trouvé en Haute-Égypte, dans les environnements du Ramesseum, l'existence (ou le dessin) d'un éléphant africain pose problème car, déjà à l'époque de l'Ancien Empire, les éléphants ont été chassés de la vallée du Nil au nord d'Assouan, autant pour des raisons de changements climatiques qu'à cause des activités humaines. Ce n'est qu'au sud d'Assouan, en Nubie, que l'espèce de l'éléphant africain (Loxodonta africana) et l'espèce, de taille plus petite, de l'éléphant de forêt d'Afrique (Loxodonta cyclotis) 7 survécurent. En Nubie, au royaume de Méroé, l'existence d'éléphants est attestée par de nombreuses représentations encore 
au $3^{\mathrm{e}}$ siècle avant notre ère. À ce moment de l'Histoire, entre 270 et environ 200 , les rois ptoléméens importèrent des éléphants de forêt du royaume de Méroé ainsi que de la côte sud de la mer Rouge et de son arrière-pays pour en faire - à l'instar des Séleucides (qui, cependant, employaient des espèces indiennes) - des éléphants de guerre $^{8}$. Les animaux capturés sous les Ptolémées furent embarqués de la côte du sud de la mer Rouge vers le nord sur des navires de transport, les " elephantaegoi ", construits tout spécialement à cette fin. En raison de la pénibilité de la voie maritime, les officiers de la chasse cherchèrent à transférer le transport des éléphants autant que possible au sud, sur la terre ferme. Ainsi, depuis 275 avant notre ère, le port naturel Berenike Troglodytica $\left(23^{\circ} 54^{\prime} \mathrm{N}\right)$ fut choisi comme lieu de transbordement principal $^{9}$. De Berenike, les éléphants étaient conduits sur une ancienne route de commerce, équipée de plusieurs relais d'eau, vers le nord-ouest, à Edfu et Koptos d'où ils étaient embarqués sur le Nil vers la Haute-Égypte.

Le long de la route de Berenike à Edfu, à l'emplacement du temple d'Al-Kanayis, on a trouvé sur une roche le graffiti, avec le dessin d'un éléphant, du charpentier Doriōn, qui était parti pour la chasse avec Eumède, entre 285 et 246 avant notre ère. Revenu en Égypte, il se réjouit de son retour : " Doriōn, un charpentier de ceux qui (étaient) chez Eumedos, parti pour la chasse aux éléphants : et je fus emmené en sécurité en Égypte ${ }^{10}$. Après la défaite de Ptolémée IV contre Antioche III dans la bataille de Raphia en 217 avant notre ère, où les éléphants africains des Ptolémées s'avérèrent inférieurs aux éléphants indiens des Séleucides, l'importation de ces animaux fut abandonnée sous Ptolémée V (205-180 av. J.-C.).

$\mathrm{Au} 3^{\mathrm{e}}$ siècle avant notre ère, beaucoup de gens devaient donc avoir participé à la chasse, au transport par eau et par terre, à l'accompagnement et au domptage des éléphants, et pour cette raison avoir eu une impression vivante de ces animaux. En même temps, pendant leur transport par terre et sur le Nil, les éléphants importés furent largement visibles par les habitants de l'Égypte, et ce pour au moins un demi-siècle. La représentation d'un éléphant au pas de course, très animé, et d'un homme avec une trompette sur l'ostracon 1298, est-elle d'une façon ou d'une autre liée à la chasse aux éléphants et à leur transport par eau ou par terre durant le $3^{\mathrm{e}}$ siècle avant notre ère ${ }^{11}$ ? La question mérite en tout cas d'être posée.

\section{La typologie de l'éléphant sur l'ostracon 1298}

Le premier général de l'Ouest à avoir pris connaissance des éléphants de guerre était Alexandre. En 331 av. J.-C., à Gaugamèles, dans la bataille contre Darius III, et en 326 dans la bataille de l'Hydaspe contre Pôros, il se trouva vis-à-vis d'éléphants indiens - et il les vainquit ${ }^{12}$. C'est entre autres sur les pièces de monnaie de l'époque des Diadoques, après 323 av. J.-C., que le souvenir de la campagne d'Alexandre en Inde est attesté : la tête d'Héraclès, jusque-là courante, est désormais remplacée par la tête d'Alexandre le Grand avec un scalp d'éléphant. Le scalp est présenté avec une trompe élevée, et ressemble quasiment à un uræus ${ }^{13}$. Par contre, sur les représentations d'éléphants vivants sur des pièces de monnaie, la trompe est toujours dirigée vers le bas ${ }^{14}$.

En 49 avant notre ère, 150 ans après l'abandon du projet " éléphants de guerre " par les Ptolémées, César fit battre ses fameux deniers en argent avec la reproduction d'un éléphant sur le verso, avançant vers la droite et, la trompe levée, piétinant d'un de ses pieds de devant un serpent qui s'est dressé contre lui. Il semble que la posture agressive du serpent soit la raison de l'attitude dynamique et la trompe levée. Les pattes arrière de l'éléphant sont parfois disposées en parallèle, pendant que les pattes de devant forment une sorte de large $\mathrm{V}$ inversé. Cette conformité de la posture de l'éléphant sur les deniers et sur l'ostracon 1298 est-elle fortuite, ou l'artiste s'est-il inspiré de la représentation de l'éléphant sur le denier de César ${ }^{15}$ ?

Là encore, l'énigme est à résoudre.

\section{Régine Hunziler-Rodewald}




\section{ORIENTATIONS BIBLIOGRAPHIQUES :}

— Casson, Lionel, « Ptolemy II and the Hunting of African Elephants ", in Transactions of the American Philological Association, 123 (1993), p. 247-26o

- Charles, Michael B., "African Forest Elephants and Turrets in the Ancient World ", in Phoenix, 62 (2008), p. 338-362

- Holt, Frank L., Alexander the Great and the Mystery of the Elephant Medallions, Berkeley, University of California press, 2003

- Meredith, David, "Berenice Troglodytica ", in The Journal of Egyptian Archaeology, 43 (1957), p. 56-70

- Murray, G. W. et Warmington, E. H., « Troglodytica: the Red Sea Littoral in Ptolemaic Times ", in The Geographical Journal, 133 (1967), p. 24-33

— Nousek, Debra L., "Turning Points in Roman History: The Case of Caesar's Elephant Denarius ", in Phoenix, 62 (2008), p. 290-307, fig. 1-11

- Scullard, Howard Hayes, The Elephant in the Greek and Roman World, Cambridge, Thames and Hudson, 1974

- Woytek, Bernhard, «Iulius Caesars Finanzen und Münzprägung im Jahre $49 \mathrm{v}$. Chr.: Bemerkungen zu RRC 443/1 ", in C. Alfaro et al. (éd.), XIII Congreso Internacional de Numismática, Madrid, Actas-proceedingactes. 2 vols., Madrid, Ministerio de Cultura, 2005, vol. 1, 2003, p. 643-648

\section{Notes}

1 - Les ostraca Aram.2 à 6 ont été publiés par Mark Lidzbarski dans Ephemeris für Semitische Epigraphik. Dritter Band 1909-1915, Giessen, Töpelmann, 1915, p. 22-28 et 298-301. Voir le carnet d'inventaire de la BNU (Espace Patrimoine), intitulé Papyrus-Sammlung IX, c) aram. Ostraka (Aram.).

2 - Information aimablement transmise par Frédéric Colin, directeur de l'Institut d'égyptologie de l'Université de Strasbourg, conservateur de la collection égyptienne de l'université et directeur de l'UMR 7044 Archimède.

3 - Wilhelm Spiegelberg, Hieratic Ostraka and Papyri found by J.E. Quibell in the Ramesseum, 1895-6, Egyptian research account, extra volume, London, Quaritch, 1898, H. 165, 167, 250, 266, 321, 323, et Yvan Koenig, Les ostraca hiératiques inédits de la Bibliothèque nationale et universitaire de Strasbourg, Le Caire, Institut français d'archéologie orientale, 1997.

4 - Sous la direction de Pascal Disdier, USR 3227, MISHA, Université de Strasbourg.

5 - Nous remercions vivement Daniel Bornemann, conservateur en charge de la Réserve de la BNU, de nous avoir accompagné et conseillé pendant nos recherches.

6 - Nous remercions les chercheurs suivants pour leur avis précieux : Ingo Kottsieper, Göttingen ; André Lemaire, Paris ; Frédéric Colin, Strasbourg ; Robert Deutsch, Jaffa/Tel Aviv; Stefan Wimmer, Munich ; Hanna Jenni, Bâle ; Julia Budka, Munich ; Jochen Hallof, Wurzbourg ; Günter Vittmann, Wurzbourg ; Philip Schmitz, Michigan ; Pierre Tallet, Paris ; Reinhard Lehmann, Mayence ; Ben Haring, Leyde ; Hans Foerster, Vienne.

7- Charles, 2008, p. 338-340, 346-349 (voir bibliographie)

8 - Casson, 1993 (voir bibliographie)

9 - Meredith, 1957 (voir bibliographie)

10-Murray/Warmington, 1967 (voir bibliographie)

11 - Seule une analyse de l'argile pourrait, au moins par la détermination d'un terminus post quem, contribuer à une clarification de l'origine et de la datation de l'inscription et des dessins sur l'ostracon 1298.

12-http://www.sylloge-nummorum-graecorum.org/ (recherche : elephant scalp), consulté le 24/04/2016

13-Emblème des pharaons, en forme de serpent naja dressé ; cf. Holt, 2003, p. 47-67 (voir bibliographie).

14- Avant le denier en argent de César (49 av. J.-C. ; Nousek, 2008, fig. 1 ; Woytek, 2003, p. 648), nous ne connaissons que très peu de pièces de monnaie avec la reproduction d'un éléphant avec la trompe levée : par ex. la tétradrachme en argent de Séleucos ${ }^{\text {er }}$, frappée aux alentours de 306 avant notre ère (Scullard, 1974 : XIII, XXIII).

15- Voir note 11. 